\title{
Modification of Tetragnatha montana (Araneae, Tetragnathidae) web architecture induced by larva of the parasitoid Acrodactyla quadrisculpta (Hymenoptera, Ichneumonidae, Polysphincta genus-group)
}

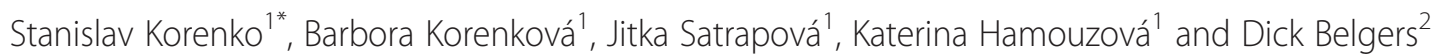

\begin{abstract}
Background: The polysphinctine wasp, Acrodactyla quadrisculpta, is a koinobiont ecto-parasitoid of spiders and is narrowly associated with the biology of its spider hosts. The larva, attached to the dorsal side of the abdomen, develops while the spider continues foraging. Shortly before pupation, the parasitoid larva manipulates the web-building activity of the host in order to construct a safe shelter against natural elements and predators during parasitoid pupation.

Results: A. quadrisculpta was associated exclusively with the orb web weaving spiders Tetragnatha montana, with a parasitism incidence of 19\%. The manipulated spider constructed a unique cocoon web that provided strong mechanical support for the parasitoid's pupal cocoon. The cocoon web consisted of one highly reinforced main thread, tensioned in $60 \%$ of cases by a reinforced side thread. The wasp cocoon, square in cross-section, was fastened along its length to the main cocoon thread.

Conclusions: The wasp A. quadrisculpta was exclusively associated with an orb-weaving spider T. montana in the family Tetragnathidae. The alteration of the web architecture of T. montana induced by the larva A. quadrisculpta was unique and species specific.
\end{abstract}

Keywords: Host-parasitoid interaction; Host manipulation; Spider host; Ephialtini

\section{Background}

Parasitic organisms have often evolved the ability to manipulate the host phenotype, including its morphology, physiology and behaviour, for their own benefit (Moore 2002). Polysphinctine wasps (the Polysphincta genus-group sensu Gauld and Dubois 2006), which are all external parasitoids of spiders, exhibit a unique trait within the Ichneumonidae in terms of development (Fitton et al. 1987). Their larva is attached to the dorsal side of the spider's opisthosoma/prosoma, where it develops while the spider continues foraging. Shortly before pupation, some of the parasitoids

\footnotetext{
* Correspondence: korenko.stanislav@yahoo.com

'Department of Agroecology and Biometeorology, Faculty of Agrobiology, Food and Natural Resources, Czech University of Life Sciences Prague, Kamýcká 129, 16521 Prague 6, Suchdol, Czech Republic Full list of author information is available at the end of the article
}

(final instar larvae) manipulate the web-spinning activity of the host in order to establish effective protection against enemies and the environment (e.g. Eberhard 2000a, 2013; Matsumoto 2009; Korenko and Pekár 2011; Korenko et al. 2014). These effects of the larva are apparently due to chemical products that are introduced into the spider (Eberhard 2010).

A few studies have been devoted to the behavioural manipulation of orb web building spiders. Eberhard (2000a, b, 2001, 2013) and Sobczak et al. (2009) studied parasitoids associated with orb web building spiders from the family Tetragnathidae; Gonzaga et al. (2010) described the manipulation of spiders from the family Nephilidae; and Gonzaga and Sobczak (2007, 2011), Eberhard (2013) and Korenko et al. (2014) studied the manipulation of spiders from the family Araneidae. The studies revealed that the manipulated spider modifies 
the architecture of its web in various ways. The orb web is modified to the 'cocoon web' (termed by Eberhard (2000a, b) for the first time) when some of its components are reduced (e.g. web spiral, radii) and others are reinforced (e.g. radii, central hub, frame) or multiplied (e.g. threads). The cocoon web is stronger and effectively designed to provide more durable support for the wasp's cocoon than the normal web (e.g. Eberhard 2000a, b). The tetragnathid spider Leucauge argyra (Walckenaer, 1841) is manipulated by the larva of Hymenoepimecis argyraphaga Gauld, 2000 to build a web which consists of a low number of radial threads radiating in a plane from a central hub; the architecture of the cocoon web remains two-dimensional (hereafter 2D) (Eberhard 2000a, b, 2001). A similar 2D cocoon web is built by the related species Leucauge roseosignata Mello-Leitão, 1943 manipulated by Hymenoepimecis japi Sobczak, Loffredo, Penteado-Dias and Gonzaga, 2009 (Sobczak et al. 2009). A similar 2D architecture of the cocoon web, but protected by the 3D structure of the tangle positioned below the hub, was recently described in the spider hosts Leucauge mariana (Keyserling, 1881) manipulated by Hymenoepimecis tedfordi Gauld, 1991 (Eberhard 2013) and Leucauge volupis (Keyserling, 1893) manipulated by Hymenoepimecis jordanensis Loffredo \& Penteado-Dias, 2009 (Gonzaga et al. 2014). In contrast, the larva of Eruga gutfreundi Gauld, 1991 induced the same host (L. mariana) to build a completely different three-dimensional cocoon web (hereafter 3D) (Eberhard 2013).

Cocoon webs of spiders from the families Araneidae and Nephilidae are mostly 3D. Three-dimensional cocoon webs are induced in araneid spider hosts in which the webs of unparasitised individuals are only 2D (Gonzaga and Sobczak 2011; Korenko et al. 2014). The cocoon web for the Acrotaphus wasp built by araneid hosts Argiope argentata (Fabricius, 1775) is 3D composed of non-sticky threads (Gonzaga and Sobczak 2011). Further, the wasps Sinarachna pallipes (Holmgren, 1860), Polysphincta tuberosa (Gravenhorst, 1829) and P. boops Tschek, 1868 manipulate spiders of the genus Araniella in a similar way (Korenko et al. 2014). All three species induced the production of a 3D structure instead of a 2D web, but thread density, thread concentration and the location of pupa on the cocoon web differed among species. The normal web of nephilid spiders consists of a 2D orb web and a 3D tangle of barrier threads at the side and its resting web is only $3 \mathrm{D}$. Both the orb and the 3D tangle of the normal web are rebuilt by the manipulated spider to form the cocoon web, whose architecture is similar to the 3D resting web (Gonzaga et al. 2010; Korenko, unpublished data). The nephilid spider Nephila clavipes (Linnaeus, 1767) was manipulated by Hymenoepimecis robertsae Gauld, 1991 and $H$. bicolor (Brulle, 1846) to build a cocoon web which consisted of a hub-like platform (part of the rebuilt orb web), from which the cocoon was suspended, and a 3D structure of non-adhesive threads of variable density. The radii and the spiral of the orb web were mostly reduced, and the wasp's cocoon was attached to the reduced orb and the barrier threads on the side (Gonzaga et al. 2010). An interesting modification of the web architecture of orb web weavers of the genera Cyclosa (Araneidae), in which the 2D orb web retains its $2 \mathrm{D}$ structure after modification (the suppression of adhesive components and a change in the radii structure), was documented in wasps of the genus Reclinervellus, which used the web stabilimentum (the structure built by the spider serving as camouflage) as the same camouflage for its cocoon (Matsumoto and Konishi 2007). The number of descriptions of web architecture alterations induced by polysphinctine final instar larvae has increased in the last few decades, but no detailed study of the web alteration induced by the Acrodactyla wasp has been performed.

We studied the interaction between the parasitoid wasp Acrodactyla quadrisculpta (Gravenhorst, 1820) and its spider host Tetragnatha montana Simon 1874 and described in detail the manipulation of web architecture induced by the parasitoid larva. A. quadrisculpta is reported from most of the Holarctic, and the species is known to be associated with the following tetragnathid spiders: T. montana, T. obtusa Koch, 1837 and T. extensa (Linnaeus, 1785) (Nielsen 1937; Fitton et al. 1988). However, very little is known about its biology and its interaction with the spider host (only Nielsen 1937 and Belgers et al. 2013).

\section{Methods}

\section{Collecting and field investigation}

The spiders (T. montana) and polysphinctine parasitoids (A. quadrisculpta) were studied in a deciduous forest close to Fondotoce di Verbania (Italy, Lake Maggiore, $\left.45^{\circ} 56^{\prime} 16^{\prime \prime} \mathrm{N}, 8^{\circ} 29^{\prime} 37^{\prime \prime} \mathrm{E}\right)$ on 30 and 31 October 2012 and in a Norway spruce stand (Picea abies) (The Netherlands, Blauwe Kamer, 51 94' 40" N, 5 61' 88" E) on 31 March 2012. Spiders were collected by beating tree canopies and undergrowth (30 to $200 \mathrm{~cm}$ above the ground) with a square-shaped beating net $\left(1-\mathrm{m}^{2}\right.$ area $)$ placed beneath. The collected specimens were fixed in $70 \%$ or pure alcohol and identified to species/genus level using Nentwig et al. (2014) and classified according to foraging guild (orb web, tangle web weavers, foliage runners, ambushers and stalkers) (Uetz et al. 1999). The spider nomenclature follows the World Spider Catalog (2014). The identification of juvenile spider hosts was confirmed by rearing spiders to adulthood (Italian specimens) and through analysis of DNA from the remains of the spider (from Dutch specimens), using the procedures described by Miller et al. (2013). Parasitoid specimens were reared to adulthood for identification in the laboratory. Adult wasps were identified using Fitton et al. 
(1988). The nomenclature of the polysphinctines follows Fitton et al. (1988) and Yu and Horstmann (1997). Voucher specimens were deposited in the personal collection of the first author and Kees Zwakhals (Netherland).

The composition of the potential host spectrum, the incidence of parasitism and the composition of the parasitoid community were recorded in the Italian locality. The incidence of parasitism was defined as the total number of cases of parasitised spiders divided by those collected by beating at the same site and time. The average parasitism incidence was the average of all three samples.

\section{Behavioural study}

Parasitised and unparasitised spiders of the genus Tetragnatha for laboratory study were collected by the same method as mentioned above. Specimens were taken alive to the laboratory for the investigation of their web building behaviour. Italian spider hosts were kept at room temperature $\left(22^{\circ} \mathrm{C} \pm 3^{\circ} \mathrm{C}\right)$ under a light/day regime of 12:12 and fed with flies Drosophila melanogaster Meigen, 1830; Drosophila hydei Sturtevant, 1921; small crickets Acheta domestica (Linnaeus, 1758); and larvae of mealworm Tenebrio molitor Linnaeus, 1758. Parasitised spiders from the Netherlands $(N=3)$ were kept at room temperature $\left(20^{\circ} \mathrm{C} \pm 2^{\circ} \mathrm{C}\right)$ under a light/day regime of 12:12 but were not fed in captivity.

The web architecture of both unparasitised spiders $(N=$ 20) and parasitised spiders ( $N=22$ from Italy, $N=3$ from the Netherlands) was analysed (web dimensionality, frequency of orb/resting web production, number of spirals and radii on orb web and its web orientation) and differences between them were identified. Spiders were placed singly into narrow plexiglass experimental arenas (frame $220 \times 220 \mathrm{~mm}$, depth $20 \mathrm{~mm}$ ) with paper tape on four sides of the frame so that the spiders could build webs, or square glass experimental arenas (base $400 \times 400 \mathrm{~mm}$, height $550 \mathrm{~mm}$ ) with a 3D construction (a cube-shaped frame with a side length of $30 \mathrm{~cm}$ ) in the middle to provide support for webs. Spiders from the Netherlands were placed separately in small glass containers $(450 \mathrm{ml}$, including a little moist moss) closed with a lid perforated with some small holes. Narrow experimental arenas where the web was built vertically were used for photo documentation, and large square arenas were used as control to recognize if there were any differences in spider behaviour between the two types of provided space (3D vs. 2D).

The web building behaviour of unparasitised spiders was recorded at 1- to 2-day intervals for 3 months. The web building behaviour of parasitised spiders was observed until the larva had consumed the spider and pupated. The web structures of both parasitised and unparasitised spiders were photographed using a Canon EOS 500 digital camera with Canon EFS 18- to 55-mm objective (Canon, Tokyo, Japan), $0.28 \mathrm{~m} / 0.9 \mathrm{ft}$, and spider behaviour under the influence of the parasitoid larva and the most important parts of the parasitoid life history were recorded using a Canon HFX 10 camcorder with a 6× Model CM-3500 35-mm microscopic lens (Canon, Tokyo, Japan).

\section{Measurement and statistics}

Measurements of thread diameters were performed using NIS Elements Documentation Software on a Zeiss Stemi SV 11 microscope (Carl Zeiss, Thornwood, NY, USA) with a Nikon DS-2Mv camera (Melville, NY, USA). The diameter of the main and the side threads of the cocoon web and the diameter of the normal web threads taken from other parts of experimental arenas (taken before the period when the manipulation of the spider behaviour appeared) were measured. Obtained data were $+1 \log$ transformed. The $T$-test was used to reveal differences in thread diameter among threads sampled from different parts of the normal and altered web. The Kolmogorov and Smirnov test (KS) was used to test whether the data were sampled from a Gaussian distribution. The Student-Newman-Keuls method (SNK) was used as a post hoc test. The DiGraphPad InStat software v. 3.06 was used.

\section{Results}

Host community and incidence of parasitism

The orb web weaver T. montana was the most abundant spider species at the investigated Italian site, representing $53.6 \%$ of all collected spider individuals (from $N=$ 349). The average incidence of parasitism of T. montana was $19.1 \%$ (Table 1). All parasitised spiders were juveniles with an average body length of $4.62 \mathrm{~mm}(N=50$, $\mathrm{SD}=1.11)$. All parasitoid wasps reared from T. montana were A. quadrisculpta $(N=9)$.

\section{Web architecture of unparasitized T. montana}

Unparasitised spiders built an orb web (Figure 1a) which was typical of those of other species of Tetragnatha. The web was oriented horizontally $(N=2)$ in the cubical arena (when 3D space was provided) or vertically $(N=18)$ when only a narrow experimental arena was provided (only 2D space was provided). In the period of satiation (no capturing activity), spiders built the 'resting web', which consisted of several scattered single threads forming a sparse tangle structure in the corner of the arena (Figure 1b). A similar 'resting web' architecture (a few scattered threads) was also observed during spider moulting in captivity $(N=2)$. The normal orb web (for capturing) was attached by anchor threads, consisting of one reinforced thread anchored at several anchoring points to the frame of the experimental arena (Figure 1c). 
Table 1 Relative spider host abundance (Ab.), average incidence of parasitism (PR) and reared wasp species

\begin{tabular}{llllll}
\hline Family & Foraging guild & $\mathbf{N}$ & Ab. (\%) & PR (\%) & Associated parasitoid \\
\hline Tetragnathidae & Orb web weavers & 187 & 53.6 & 19.1 & Acrodactyla quadrisculpta \\
Araneidae & Orb web weavers & 6 & 1.7 & $?$ & Zatypota picticollis \\
Theridiidae & Tangle web weavers & 63 & 18.1 & 23.5 & Zatypota percontatoria \\
Anyphaenidae & Foliage runners & 41 & 11.7 & 0 & - \\
Clubionidae & Foliage runners & 8 & 2.3 & 0 & - \\
Thomisidae & Ambushers & 29 & 8.3 & 0 & - \\
Philodromidae & Ambushers & 8 & 2.3 & 0 & - \\
Salticidae & Stalkers & 7 & 2.0 & 0 & 12.5 \\
Total & & 349 & 100 &
\end{tabular}

? - incidence of parasitism was not calculated because of the low number of collected spiders.

\section{Interaction with parasitoid larva}

The wasp's final instar larva induced subsequent unique changes in the webbing behaviour of $T$. montana (Table 2). The spider under manipulation built a specific cocoon web $(N=12)$. In all cases, the cocoon web contained one strong horizontally oriented thread - the 'main thread'; in $60 \%$ of cases, this thread was tensioned by a 'side thread' (Figure 1d). Both threads of the cocoon web were reinforced 50 to 60 times by a layer of silk (Additional file 1: Video 1, s 01). The building of the cocoon web started 8 to 9 h before the larva killed the spider host and began to suck it dry $(N=3)$. When the modified web was finished, the spider took a position at approximately the middle of the main thread close to
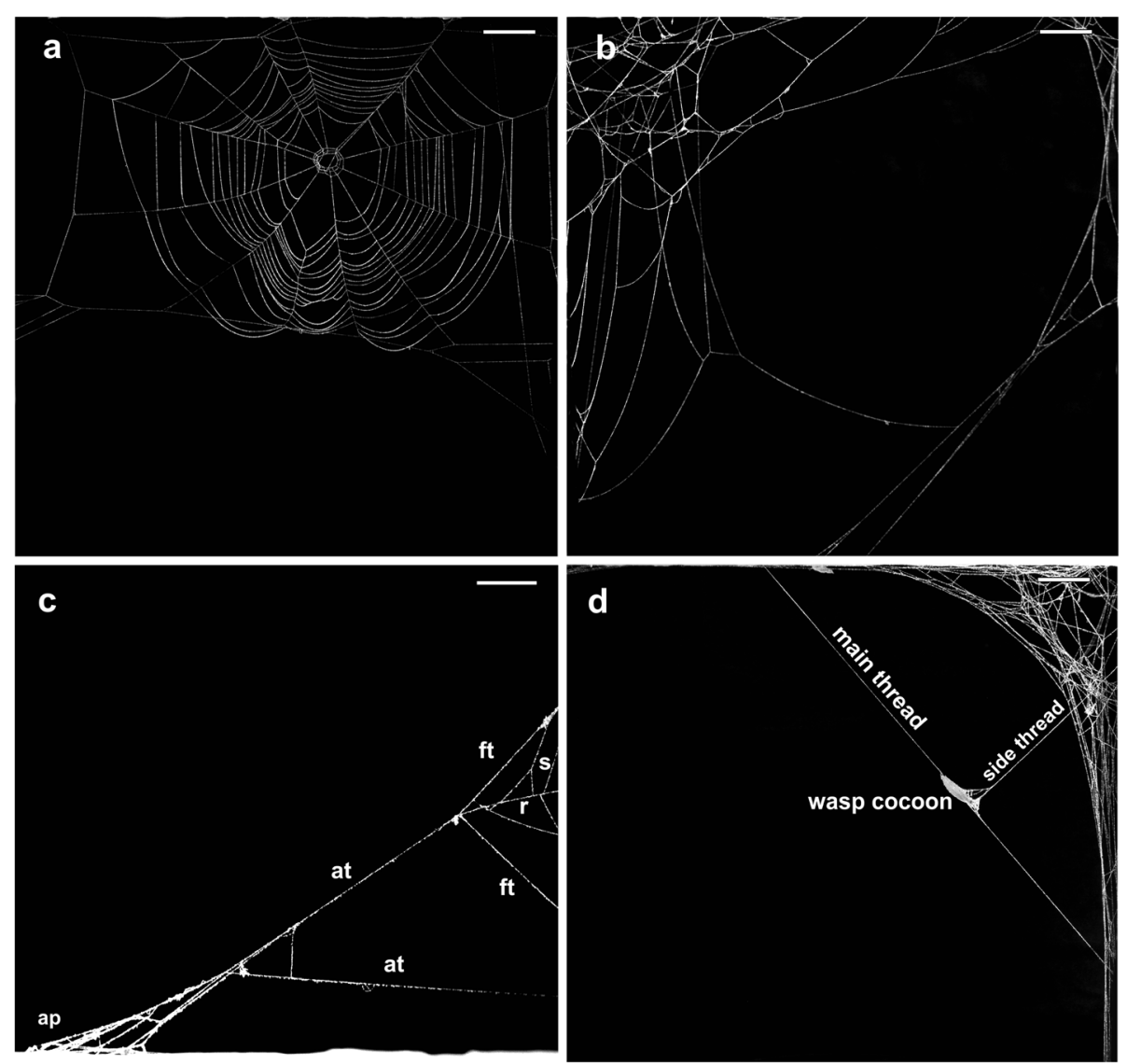

Figure 1 Normal orb web and induced cocoon web. Normal hunting orb web of T. montana (a). Resting web when orb web was not presented (b). Detail of anchoring of normal orb web (ap, anchor point; at, anchor thread; $\mathrm{ft}$, frame thread; $\mathrm{r}$, radial thread; s, spiral) (c). Induced cocoon web consisting of the main thread and side thread (d). Scale $=20 \mathrm{~mm}$ (a, b), $5 \mathrm{~mm}$ (c) and $10 \mathrm{~mm}$ (d). 
Table 2 Behaviour table of host-parasitoid interactions in laboratory

\begin{tabular}{|c|c|c|c|}
\hline Day & Time & Observed behaviour & Video \\
\hline 1st (30 December 2012) & 21:00 & Changes in spider behaviour; the spider was very active, webbing in several places of the experimental arena. & - \\
\hline \multirow[t]{5}{*}{ 2nd } & 3:00 & $\begin{array}{l}\text { One thread was chosen by the spider. The thread was reinforced } 58 \text { times during the next } 2 \mathrm{~h} \text {. The spider } \\
\text { rested, suspended on the thread or at the place where the thread was attached to the arena frame during } \\
\text { the spinning of each silk layer. }\end{array}$ & s 01 \\
\hline & 5:15 & $\begin{array}{l}\text { The spider took a position in the middle of the main thread of the cocoon web and died. The larva } \\
\text { attached itself to the main thread by its dorsal tubercles. The larva began to consume the spider. }\end{array}$ & s 02 \\
\hline & 10:40 & $\begin{array}{l}\text { The spider was completely consumed. The spider carcass was dropped onto the ground. The larva rested, } \\
\text { suspended on the main thread of the cocoon web. }\end{array}$ & s 03 \\
\hline & $12: 00$ & The larva started to build a cocoon for pupation. & s 04 \\
\hline & 20:00 & $\begin{array}{l}\text { The outer layer of the cocoon was finished, and the larva closed itself inside the cocoon. The larva spans the } \\
\text { inner layers of the cocoon wall. }\end{array}$ & s 05 \\
\hline $3 r d$ & 20:00 & The larva finished the cocoon. After which, the larva exhibited low activity. & s 06 \\
\hline 4th & $8: 00$ & The cocoon was completely finished, and the larva displayed no further activity. & s 06 \\
\hline 15th & 6:00 & The adult wasp emerged from the cocoon. & s 07 \\
\hline
\end{tabular}

the connection with the side thread if it was present and grasped the main thread with all its legs until it died (Additional file 1: Video 1, s 02). The larva, still attached to the opisthosoma of the already dead spider, hung itself on the main thread using its dorsal tubercles, preventing itself from falling to the ground (Additional file 1: Video 1, s 03). Seven pairs of dorsal tubercles appeared after the spider manipulation began. Tubercles were retracted repeatedly. When the spider was completely sucked dry and the spider remains were eventually discarded, the parasitoid larva (having almost doubled its size) took a position on the 'main thread' connected to it by its dorsal tubercles. Over the next $30 \mathrm{~h}$, the larva spans its cocoon and later pupated inside (Additional file 1: Video 1, s 04-06). The cocoons enclosing pupae were fusiform, square in cross-section, and from white to yellowish brown (depending on the level of humidity in the experimental arena). The cocoon's long axis was along the axis of the main thread of the cocoon web. The cocoon walls were densely woven with a tight and smooth outer surface.

Adult wasps emerged after 10.2 days $(\mathrm{SD}=0.75, N=6)$ in Italy and 13.7 days $(\mathrm{SD}=0.57, N=3)$ in the Netherlands (Additional file 1: Video 1, s 07). The sex ratio of the reared wasps was 1:2 (female:male) in both Italy and the Netherlands.

\section{Reinforcement of threads}

The diameter of the main threads of the cocoon web was on average $21 \mu \mathrm{m}(\mathrm{SD}=9.735, N=8)$. It did not differ significantly to the diameter of the side threads of the cocoon web, which had an average diameter of $24 \mu \mathrm{m} \quad\left(\mathrm{SD}=8.734, N=8 ; t_{14}=0.488, p=0.63\right)$. The diameter of normal web threads, taken from other parts of experimental arenas, averaged $<5 \mu \mathrm{m}(\mathrm{SD}=0.417, N=8)$ and was significantly thinner than both the main threads and side threads of the cocoon web $\left(t_{14}=4.888, p=0.0002\right.$ and $t_{14}=6.150, p<0.0001$ ) (Figure 2). All data groups passed the KS normality test. Variation between the group of control threads and groups of cocoon web threads was significantly greater than that expected by chance (SNK, $p<0.05)$. Variation between the group of main threads and the group of side threads was not significant (SNK, $p<0.05)$. The reinforcement of the main and side threads of the cocoon web was achieved by repeatedly adding silk lines on the already existing thread (Additional file 1: Video 1, s 01). The architecture of the anchoring of the cocoon webs (Additional file 1: Video 1, s 01) was similar to the anchoring of the frame threads in normal orb webs (for capturing) (Figure 1c). Both consisted of one

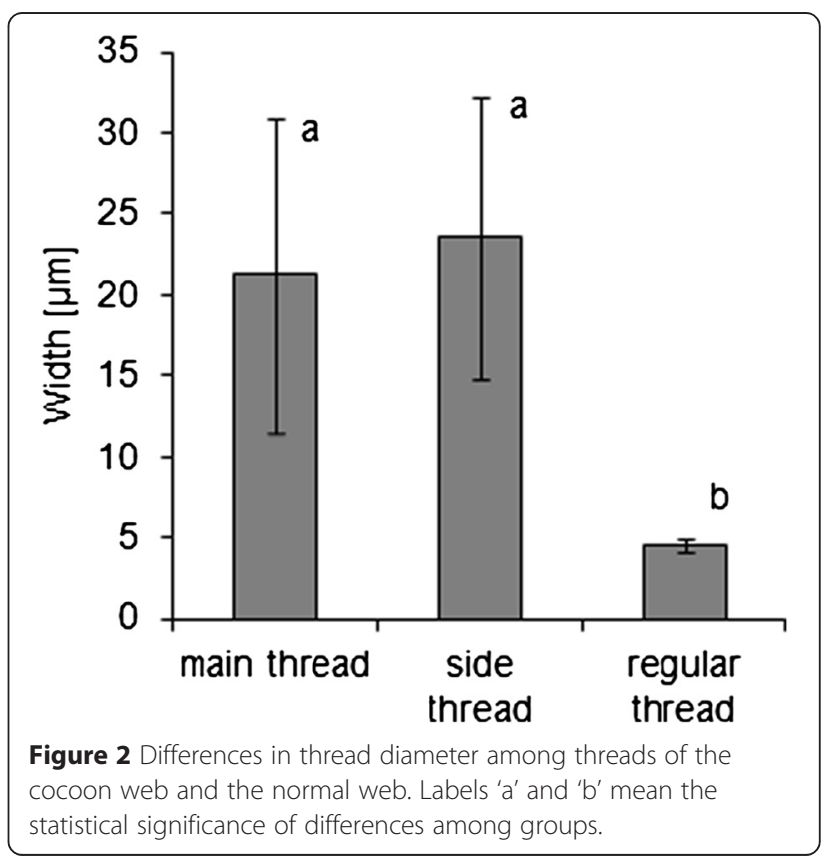


reinforced thread anchored at several anchoring points to the frame of the experimental arena.

\section{Discussion}

We found that the wasp $A$. quadrisculpta was the most abundant spider parasitoid in the samples from the studied locality and was exclusively associated with the abundant web building spider T. montana, which represented more than $50 \%$ of the spiders in the samples. A. quadrisculpta is thought to be exclusively associated with spider hosts of the genus Tetragnatha (e.g. Nielsen 1937; Fitton et al. 1987, 1988; this study). Although araneid spiders of the genus Araniella were assumed to be hosts of A. quadrisculpta by Nielsen (1937), clear evidence linking it to this host is missing. Korenko et al. (2014) reported that all parasitoids reared on spiders of the genus Araniella collected during 4 years of study in Italy belonged to the genera Sinarachna and Polysphincta. On the basis of this fact, we suspect that A. quadrisculpta is capable of successfully attacking only spiders of the genus Tetragnatha, which includes several species with similar morphological, ecological and behavioural patterns. Polysphinctine wasps in Europe seem to be associated with species occurring abundantly (at least locally) (e.g. Korenko et al. 2011, 2014; Korenko et al., unpublished data). This was also observed in the studied wasp A. quadrisculpta. The association with abundant species seems to be effective and could be a consequence of a narrow host spectrum and the high specificity of the evolved adaptations to capture the particular spider host. We assume that the association with rare species, or species with low abundance in the community of potential hosts, could be leading to the extinction of the parasitoid population, because females, which do not find a sufficient number of suitable spider hosts, are not able to establish the next generation.

The final instar larva of A. quadrisculpta induced unique changes in spider web architecture that consisted of only one strong main thread, often but not always tensioned by one additional lateral thread supporting the wasp's cocoon. Similar cocoon webs, which consisted of only a few (sometimes only two) threads, were also observed in other tetragnathid spiders: L. argyra manipulated by $H$. argyraphaga (Eberhard 2000a) and L. roseosignata Mello-Leitão, 1943 manipulated by $H$. japi Sobczak et al., 2009 (Sobczak et al. 2009). Eberhard (2000a) described that the construction of the cocoon web induced by $H$. argyraphaga was nearly identical to the early steps in one subroutine of normal orb construction of L. argyra, and the other normal orb construction behaviour patterns were mostly repressed (Eberhard 2001). The cocoon web induced had, in most cases, no true hub (with hub loops) but rather non-spiral lines at the convergence of the radius lines, no frame lines and usually no tangle; however, in extremely reduced cases, the cocoon web consisted of only a single strong line with the cocoon suspended from the central portion (Eberhard 2000a, b, 2001; Eberhard, personal communication). These, the simplest observed cocoon webs induced by $H$. argyraphaga, had a general appearance similar to the cocoon web of $A$. quadrisculpta (one strong thread), but differed in several respects. The cocoon of A. quadrisculpta was horizontal, placed longitudinally along the horizontally oriented main thread. The main thread was present in all cocoon webs. In contrast, the cocoon of $H$. argyraphaga was suspended vertically on a line that was attached at the central point where the radial lines converged. The final architecture of the cocoon web induced by $H$. argyraphaga was more varied (Eberhard 2000a, b, 2001). In our study, the main thread of the cocoon web appeared to be morphologically and functionally similar to the frame thread of the normal orb web; both were strong structures that supported other parts of the web, such as the sticky spiral of the capture web (Additional file 1: Video 1, s 01) (Figure 1c).

The pupal cocoon of A. quadrisculpta is square in cross-section similar to those of some other polysphinctine wasps of the genera Acrodactyla (Fitton et al. 1987) and Eruga (Eberhard 2013). The morphology and position of the pupal cocoon are often species/genus/taxonomical group specific (e.g. Fitton et al. 1988; Matsumoto and Takasuka 2010; Korenko et al. 2014). This can be useful in the identification of wasp species, at least to genus level (Korenko et al. 2014; Korenko, unpublished data). In contrast, the coloration of cocoons varies. In our study, pupa cocoons of $A$. quadrisculpta placed in a dry environment were snow white (Additional file 1: Video 1, s 06), but cocoons in an environment with high humidity became orange-brown in colour (Additional file 1: Video 1, s 07).

\section{Conclusions}

The studied wasp A. quadrisculpta was associated only with the spider T. montana. This wasp species seems to be exclusively associated with orb web weaving spiders of the genus Tetragnatha from the family Tetragnathidae. The unique behavioural manipulation induced by the larvae of $A$. quadrisculpta was described for the first time. The architecture of the cocoon web induced by a particular wasp is species specific.

\section{Additional file}

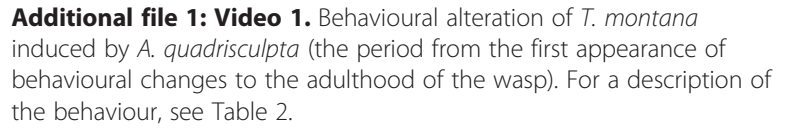

Competing interests

The authors declare that they have no competing interests. 


\section{Authors' contributions}

SK carried out the collection of Italian specimens, conducted behavioural observations in the laboratory, participated in the video recording and photography, wrote the main text of the manuscript and participated in the statistical analysis. BK participated in the video recording and behavioural observations. JS carried out the laboratory rearing of animals and helped to draft the manuscript. KH participated in the statistical analysis. DB carried out the collection of Dutch specimens, conducted behavioural observations and helped to draft the manuscript. All authors read and approved the final manuscript.

\section{Acknowledgements}

The study was supported by the European Science Foundation and Ministry for Education, Youth and Sport of the Czech Republic, project CZ.1.07/2.3.00/ 30.0040, and the Institutional Support Program for Long Term Conceptual Development of Research Institutions provided by the Ministry for Education, Youth and Sport of the Czech Republic.

\section{Author details}

${ }^{1}$ Department of Agroecology and Biometeorology, Faculty of Agrobiology, Food and Natural Resources, Czech University of Life Sciences Prague, Kamýcká 129, 16521 Prague 6, Suchdol, Czech Republic. ${ }^{2}$ Wageningen University, Droevendaalsesteeg 4, 6708 PB, Wageningen, Netherlands.

Received: 18 July 2014 Accepted: 29 April 2015

Published online: 14 May 2015

\section{References}

Belgers D, Zwakhals K, van Helsdingen P (2013) De bijzondere levensloop van de sluipwesp Acrodactyla quadrisculpta op de schaduwstrekspin Tetragnatha montana (Hymenoptera: Ichneumonidae, Araneae: Tetragnathidae). Nederl Faun Mededel 39:1-6

Eberhard WG (2000a) Spider manipulation by a wasp larva. Nature 406:255-256

Eberhard WG (2000b) The natural history and behavior of Hymenoepimecis argyraphaga (Hymenoptera: Ichneumonidae) a parasitoid of Plesiometa argyra (Araneae: Tetragnathidae). J Hymenopt Res 9:220-240

Eberhard WG (2001) Under the influence: webs and building behaviour of Plesiometa argyra (Araneae, Tetragnathidae) when parasitised by Hymenoepimecis argyraphaga (Hymenopera, Ichneumonidae). J Arachnol 29:354-366

Eberhard WG (2010) Recovery of spiders from the effects of parasitic wasps: implications for fine-tuned mechanisms of manipulation. Anim Behav 79:375-383

Eberhard WG (2013) The polysphinctine wasps Acrotaphus tibialis, Eruga ca. gutfreundi, and Hymenoepimecis tedfordi (Hymenoptera, Ichneumonidae, Pimplinae) induce their host spiders to build modified webs. Ann Entomol Soc Am 106:652-660

Fitton MG, Shaw MR, Austin AD (1987) The Hymenoptera associated with spiders in Europe. Zool J Linn Soc-Lond 90:65-93

Fitton MG, Shaw MR, Gauld ID (1988) Pimpline ichneumon-flies. Handbooks for the Identification British Insects 7:1-110

Gonzaga MO, Sobczak JF (2007) Parasitoid-induced mortality of Araneus omnicolor (Araneae, Araneidae) by Hymenoepimecis sp. (Hymenoptera, Ichneumonidae) in southeastern Brazil. Naturwissenschaften 94:223-227

Gonzaga MO, Sobczak JF (2011) Behavioral manipulation of the orb-weaver spider Argiope argentata (Araneae: Araniedae) by Acrotaphus chedelae (Hymenoptera: Ichneumonidae). Entomol Sci 14:220-223

Gonzaga MO, Sobczak JF, Penteado-Dias AM, Eberhard WG (2010) Modification of Nephila clavipes (Araneae Nephilidae) webs induced by the parasitoids Hymenoepimecis bicolor and $\mathrm{H}$. robertsae (Hymenoptera Ichneumonidae). Ethol Ecol Evol 22:151-165

Gonzaga MO, Moura RR, Pêgo PT, Bang DL, Meira FA (2014) Changes to web architecture of Leucauge volupis (Araneae: Tetragnathidae) induced by the parasitoid Hymenoepimecis jordanensis (Hymenoptera: Ichneumonidae). Behaviour, available online. doi:10.1163/1568539X-00003238

Korenko S, Pekár S (2011) A parasitoid wasp induces overwintering behaviour in its spider host. PLoS One 6:e24628

Korenko S, Michalková V, Zwakhals K, Pekár S (2011) Host specificity and temporal and seasonal shifts in host preference of a web-spider parasitoid (Hymenoptera: Ichneumonidae). J Insect Sci 11:101
Korenko S, Isaia M, Satrapová J, Pekár S (2014) Parasitoid genus-specific manipulation of orb-web host spiders (Araneae, Araneidae). Ecol Entomol 39:30-38

Matsumoto R (2009) "Veils" against predators: modified web structure of a host spider induced by an ichneumonid parasitiod, Brachyzapus nikkoensis (Uchida) (Hymenoptera). J Insect Behav 22:39-48

Matsumoto R, Konishi K (2007) Life histories of two ichneumonid parasitoids of Cyclosa octotuberculata (Araneae), Reclinervellus tuberculatus (Uchida) and its new sympatric congener (Hymenoptera: Ichneumonidae: Pimplinae). Entomol Sci 10:267-278

Matsumoto R, Takasuka K (2010) A revision of the genus Zatypota Förster of Japan, with descriptions of nine new species and notes on their hosts (Hymenoptera: Ichneumonidae: Pimplinae). Zootaxa 2522:1-43

Miller JA, Belgers JDM, Beentjes KK, Zwakhals K, van Helsdingen P (2013) Spider host (Arachnida, Araneae) and wasp parasitoids (Insecta, Hymenoptera, Ichneumonidae, Ephialtini) matched using DNA barcodes. Biodivers Data J 1:e992

Moore J (2002) Parasites and the behavior of animals. Oxford University Press, Oxford

Nentwig W, Blick T, Gloor D, Hänggi A, Kropf C (2014) Spiders of Europe. http://www.araneae.unibe.ch. Accessed on 20 Dec 2014.

Nielsen E (1937) A fourth supplementary note upon the life histories of the Polysphinctas (Hym. Ichneum.). Entomol Meddel 20:25-28

Sobczak JF, Loffredo APS, Penteado-Dias AM, Gonzaga MO (2009) Two new species of Hymenoepimecis (Hymenoptera: Ichneumonidae: Pimplinae) with notes on their spider hosts and behaviour manipulation. J Nat Hist 43:2691-2699

Uetz GW, Halaj J, Cady AB (1999) Guild structure of spiders in major crops. J Arachnol 27:270-280

World Spider Catalog (2014) World Spider Catalog. Natural History Museum Bern. http://wsc.nmbe.ch, version 15.5. Accessed 20 Dec 2014.

Yu DS, Horstmann K (1997) A catalogue of world Ichneumonidae (Hymenoptera). Mem Am Entomol Inst 58:1-1558

\section{Submit your manuscript to a SpringerOpen ${ }^{\odot}$ journal and benefit from:}

- Convenient online submission

Rigorous peer review

- Immediate publication on acceptance

- Open access: articles freely available online

- High visibility within the field

- Retaining the copyright to your article

Submit your next manuscript at springeropen.com 